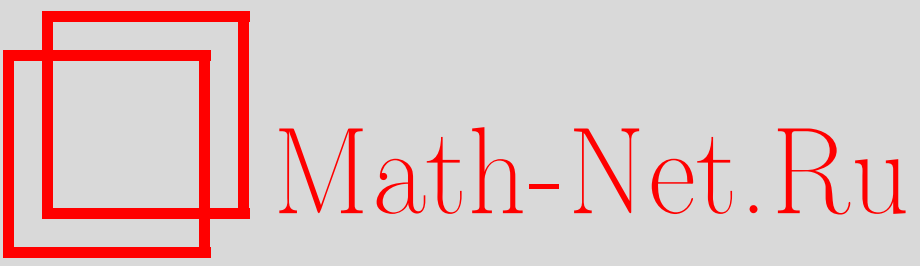

С. Ю. Славянов, Структурная теория специальных функций, ТМФ, 1999, том 119, номер 1, 3-19

DOI: https://doi.org/10.4213/tmf723

Использование Общероссийского математического портала Math-Net.Ru подразумевает, что вы прочитали и согласны с пользовательским соглашением

http://www . mathnet.ru/rus/agreement

Параметры загрузки:

IP: 54.237 .206 .68

26 апреля 2023 г., $15: 27: 40$ 
ТЕОРЕТИЧЕСКАЯ

И МАТЕМАТИЧЕСКАЯ

ФИЗИКА

Том 119, № 1

апрель, 1999

(C) 1999 г.

\author{
С. Ю. Славянов*
}

\title{
СТРУКТУРНАЯ ТЕОРИЯ СПЕЦИАЛЬНЫХ ФУНКЦИЙ
}

Предложена структурная схема для описания дифференциальных уравнений, решения которых являются специальными функциями математической физики. Выделены три класса таких уравнений: гипергеометрический, Гойна и Пенлеве. В каждом классе перечислены составляющие его типы уравнений. Описаны процессы конфлюэнции, переводящие один тип в другой. Указаны взаимосвязи между уравнениями, принадлежащими разным классам. Так, уравнения класса Пенлеве являются уравнениями классического движения для гамильтонианов, отвечающих уравнениям класса Гойна, а линеаризация уравнений класса Пенлеве приводит к уравнениям гипергеометрического класса. Сформулирован "принцип конфоюэнции” и дан пример его применения.

\section{1. ВВЕДЕНИЕ}

В данной статье предлагается вариант систематизации описания специальных функций математической физики, возникающих в качестве решений определенных обыкновенных дифференциальных уравнений (ОДУ) второго порядка, линейных и нелинейных, полиномиально зависящих от зависимой и независимой комплексных переменных. Имеется большое количество книг, посвяшенных специальным функциям, однако в большинстве из них изложение строится вокруг отдельно взятой специальной функции $[1,2]$, и только в немногих делается попытка систематизации [3-5]. С другой стороны, нам известна только одна книга [6], где "нелинейные" специальные функции (решения нелинейных ОДУ) рассмотрены наравне с “линейными” (решениями линейных уравнений).

Изложение будет сконцентрировано вокруг тех специальных функций, которые возникают как решения уравнений ${ }^{1)}:$ 1) гипергеометрического класса, 2) класса Гойна, 3) класса Пенлеве.

\footnotetext{
1) Не будучи поклонником философских теорий, автор тем не менее неожиданно для себя узнал, что разработанная им систематизация имеет вид триад, типичный для раздела философии семиотики [7]. Каждая триада состоит из трех видов объектов, для которых определены некоторые операции. Указанные операции могут включать в себя объекты из разных триад. Предложенные триады (не всегда только они) образуют иерархическое дерево, которое и является основой систематизационной модели (см. рисунок).
}

\footnotetext{
* Санкт-Петербургский государственный университет, Санкт-Петербург, Россия
} 


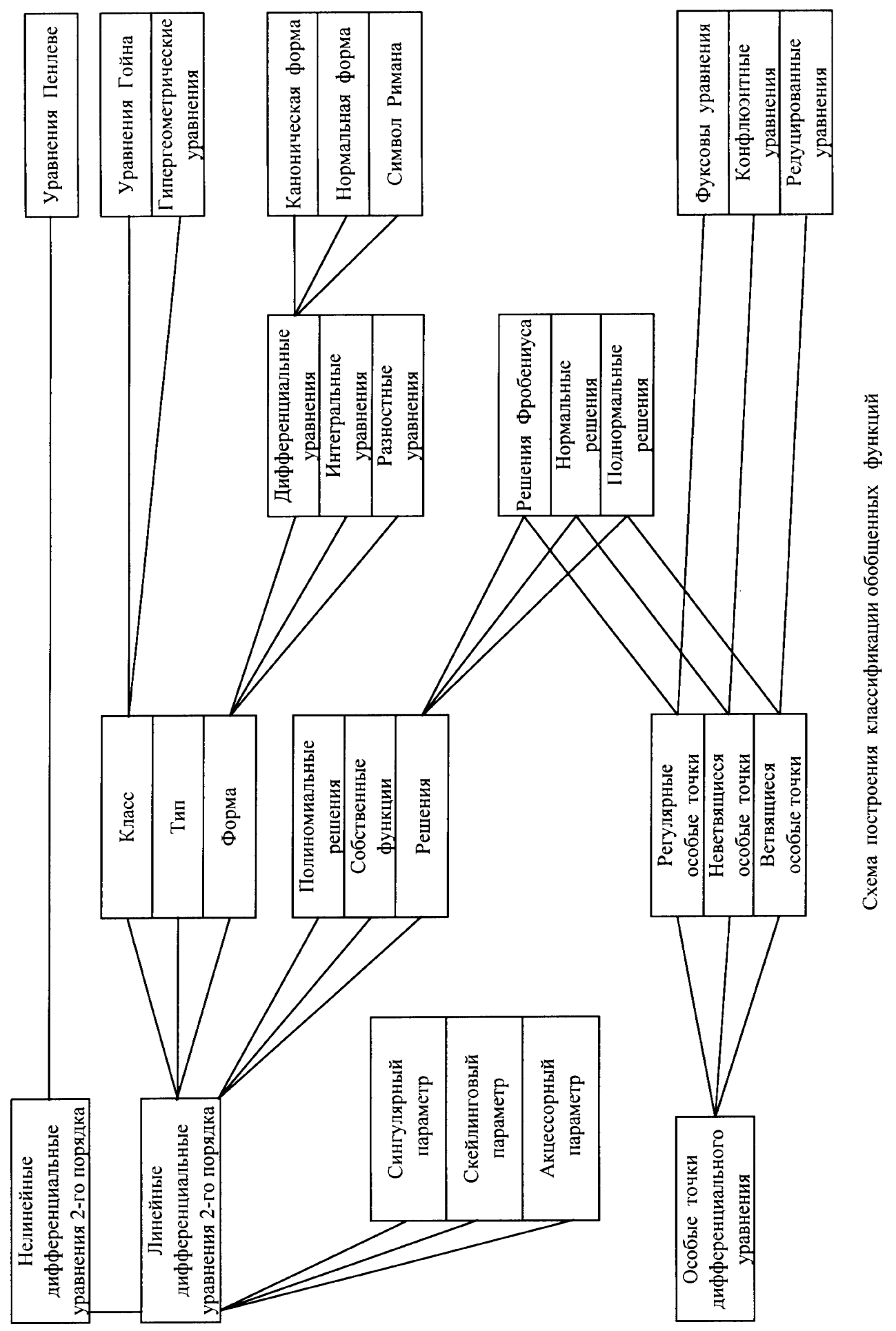


Первые два класса составляют линейные уравнения, порожденные в смысле, который будет описан ниже, гипергеометрическим уравением (фуксовым уравнением с тремя особыми точками) и уравнением Гойна (фуксовым уравнением с четырьмя особыми точками), соответственно. Третий класс - нелинейные уравнения, которые характеризуются свойством Пенлеве: подвижные особые точки решений этих уравнений могут быть только полюсами. Понятие класса линейных ОДУ второго порядка, равно как и другие понятия, используемые во введении, можно найти в статье [8]. Частично эти понятия переопределены в тексте данной статьи.

Между уравнениями, принадлежашими к указанным трем классам, устанавливаются следуюшие соотношения (возможны исключения):

1) добавление особой точки в уравнение гипергеометрического класса переводит его в класс Гойна;

2) уравнение классического движения для гамильтониана, отвечающего уравнению класса Гойна, принадлежит классу Пенлеве;

3) линеаризация уравнения из класса Пенлеве при определенном значении входяшего в него параметра переводит его в уравнение гипергеометрического класса.

\section{2. ОСОБЫЕ ТОЧКИ ЛИНЕЙНЫХ ОДУ ВТОРОГО ПОРЯДКА}

Введение классов уравнений (гипергеометрического и Гойна) основывается на классификации характеризуюших линейное ОДУ второго порядка особых точек. Мы придерживаемся классификациии, введенной в статье [8] и основанной на понятии s-ранга.

Рассмотрим линейные ОДУ второго порядка

$$
P_{0}(z) y^{\prime \prime}(z)+P_{1}(z) y^{\prime}(z)+P_{2}(z) y(z)=0
$$

с полиномиальнысми коэффициентами $P_{0}(z), P_{1}(z), P_{2}(z)$. Сингулярностями (особыми точками) уравнения (1) являются нули полинома $P_{0}(z)$ - точки $z_{j}(j=1, \ldots, n)$ и, возможно, точка $z=\infty$. Каждая сингулярность уравнения может быть охарактеризована численной величиной - s-рангом.

ОПРЕДЕЛЕНИЕ 1 . В конечной иррегулярной точке $z_{j}$ s-ранг $R_{j}$ вычисляется по формуле

$$
R\left(z_{j}\right)=\max \left(K_{1}\left(z_{j}\right), \frac{K_{2}\left(z_{j}\right)}{2}\right),
$$

где $K_{1}\left(z_{j}\right)$ - кратность нуля функции $P_{0}(z) / P_{1}(z)$ в точке $z_{j}$, а $K_{2}\left(z_{j}\right)$ - кратность нуля функции $P_{0}(z) / P_{2}(z)$ в той же точке. В бесконечной иррегулярной точке $z=\infty$ s-ранг $R(\infty)$ вычисляется путем перевода ее дробно-линейным преобразованием в конечную; s-ранг иррегулярной точки есть целое или полуцелое число больше единишы.

ОПРЕДЕЛЕНИЕ 2. Положим s-ранг регулярной особой точки равным единице, что согласуется с определениями (1)-(2). 
ЗАмечАниЕ 1. Можно показать, что приведенные определения в терминах коэффициентов уравнения эквивалентны сушествованию неулучшаемой оценки решений в окрестности особой точки

$$
\left|(\ln y(z))^{\prime}\right| \leqslant K\left|\left(z-z_{j}\right)\right|^{-R\left(z_{j}\right)-\epsilon}
$$

где $\epsilon$ - произвольное малое положительное число и $K>0$.

ЗАмЕЧАнИЕ 2. Вопрос о существовании сингулярностей с s-рангом, равным $1 / 2$, может решаться по-разному. Можно считать, что таких сингулярностей не сушествует. Если постулировать, что такие точки есть, то возникает несколько альтернативных способов их введения: либо формально применять формулу (2), либо вводить данный ранг как ранг элементарных точек, либо другими альтернативными способами. Здесь для упрощения структуры считается, что точек с s-рангом, равным $1 / 2$, нет.

Определение s-ранга $R\left(z_{i}\right)$ отличается от определения ранга особой точки, введенного Пуанкаре $R_{\mathrm{P}}\left(z_{i}\right)$, и от определения рода особой точки, введенного Айнсом $R_{\mathrm{S}}\left(z_{i}\right)$. Сравнение трех числовых характеристик особых точек дано в табл. 1.

Таблица 1

Числовые характеристики особых точек

\begin{tabular}{|c|c|c|c|}
\hline & Регулярные & \multicolumn{2}{|c|}{ Иррегулярные особые точки } \\
\cline { 3 - 4 } & особые точки & неветвящиеся & ветвяшиеся \\
\hline$R_{\mathrm{P}}\left(z_{i}\right)=$ Р-ранг & 0 & $n-1$ & - \\
$R_{\mathrm{S}}\left(z_{i}\right)=$ род & 0 & $2 n-2$ & $2 n-3$ \\
$R\left(z_{i}\right)=$ s-ранг & 1 & $n$ & $n-1 / 2$ \\
\hline
\end{tabular}

Согласно введенным определениям особые точки подразделяются следующим обра30м:

1) регулярные особъе точки с s-рангом, равным единице;

2 ) иррегулярные особые точки с целым s-рангом (неветвящиеся иррегулярные особенности),

3) иррегулярные особые точки с полуцелым s-рангом (ветвящиеся иррегулярные особенности).

В окрестности каждой из перечисленных точек специальные решения уравнения могут быть соответственно представлены:

1) в форме Фробениуса, т.е. в виде ряда, умноженного на степенную функцию;

2) в нормальной форме асимптотического разложения (в виде экспоненты от полинома, умноженной на асимптотический ряд по целым степеням);

3) в поднормальной форме асимптотического разложения (и в экспоненте и в степенных разложениях содержатся полуцелые степени).

Указанные представления при специальных значениях параметров могут также содержать логарифмы. 


\section{3. КЛАССИФИКАЦИЯ ЛИНЕЙНЫХ ОДУ ВТОРОГО ПОРЯДКА}

Набор s-рангов особых точек данного уравнения образует его s-мультисимвол. В соответствии с классификацией особых точек уравнения с полиномиальными коэффициентами могут быть разделены на следующие группы:

1) фуксовы уравнения (только с регулярными особыми точками, пример - гипергеометрическое уравнение);

2) конфлюэнтные уравнения, у которых иррегулярные особые точки имеются и могут быть только с целым s-рангом (пример - вырожденное ${ }^{2)}$ (конфлюэнтное) гипергеометрическое уравнение);

3) редуцированные конфлюэнтные уравнения, где обязательно есть иррегулярные особые точки с полуцелым s-рангом (пример - уравнение Эйри).

Отметим, что s-мультисимвол фуксова уравнения состоит из единиц, s-мультисимвол конфлюэнтного уравнения - из целых чисел, хотя бы одно из которых обязательно больше единицы, s-мультисимвол конфлюэнтного уравнения содержит хотя бы одно полуцелое число.

Линейные ОДУ второго порядка различаются по принадлежности: 1) к классу, 2) к muпу и 3) к форме.

Класc - наиболее общий систематизационньй объект, характеризуемый номером $N$ и включаюший в себя уравнения, сумма s-рангов которых удовлетворяет неравенствам

$$
N-1 \leqslant \sum_{j} R\left(z_{j}\right) \leqslant N
$$

При этом, если слева выполняется точное равенство, то для принадлежности уравнения к классу хотя бы один s-ранг этого уравнения должен быть полуцелым. Для уравнений, принадлежащих к классу, существует порождающее этот класс фуксово уравнение с $N$ регулярными особыми точками. По имени этого уравнения в простейших случаях первых значений $N$ и называется класс (гипергеометрический и т.д.).

K одному типу относятся уравнения, у которых s-мультисимвол одинаков. Таким образом, тип уравнения задается его мультисимволом. Лингвистическое определение типа достаточно вольно задается терминами конфлюэнтный, редуцированный и указанием кратности.

Уравнения заданного типа сохраняют этот тип под действием калибровочных преобразований зависимой переменной, сохраняющих число особых точек и их s-ранг, s-гомотопных преобразований [8] и дробно-линейных преобразований независимой переменной. Возникающие эквивалентные уравнения являются формами данного типа.

Из параметров, характеризующих уравнения с полиномиальными коэффициентами, выделяются следующие:

\footnotetext{
2) Прочно вошедший в отечественную литературу по специальным функциям термин "вырожденное" представляется автору крайне неудачным. В этой статье используется термин "конффлюэнтное". По своей логике термин "вырожденное" больше отвечает используемому здесь понятию "редуцированное".
} 
1) сингулярные параметры, характеризующие в сушественном поведение решения в окрестности особой точки и не меняюшиеся при масштабировании независимой переменной. Они задают показатели степеней для анзаца в окрестности особой точки;

2) скейлинговые параметры, характеризуюшие в сушественном поведение решения в окрестности особой точки и меняюшиеся при масштабировании независимой переменной. Они задают коэффициенты для полинома в экспоненте;

3) акиессорные параметры, характеризующие глобальные свойства решений и не входящие в главные члены явных локальных формул.

Решения рассматриваемых уравнений (специальные функции) подразделяются на:

1) локальные решения (решения, заданные поведением в особой точке);

2) собственнье функиии;

3) квазиполиномиальные решения.

\section{4. ГИПЕРГЕОМЕТРИЧЕСКИЙ КЛАСС}

Гипергеометрический класс $(N=3)$ порождается уравнением Римана - фуксовым уравнением с тремя регулярными особыми точками. Поместив эти особые точки с помощью дробно-линейного преобразования в точки $0,1, \infty$ и сделав с помощью s-гомотопного преобразования один из характеристических показателей в точках 0,1 равным нулю, мы переведем уравнение Римана в каноническую естественную форму [8] - гипергеометрическое уравнение, которое еше называют уравнением Гаусса,

$$
L_{z}^{(1,1 ; 1)}(a, b ; c) y(z)=\left(z(1-z) D^{2}+(c-(a+b+1) z) D-a b\right) y(z)=0, \quad D=\frac{d}{d z}
$$

Локальные свойства этого уравнения отражаются обобшенной схемой Римана

$$
\left(\begin{array}{cccc}
1 & 1 & 1 \\
0 & 1 & \infty & ; z \\
0 & 0 & a \\
1-c & c-a-b & b
\end{array}\right)
$$

B первой строке приведены s-ранги особых точек, во второй строке - их местоположение, в третьей и четвертой строках - характеристические показатели решений Фробениуса, построенных в этих точках.

Перечислим пять типов уравнений, входящих в гипергеометрический класс начиная с уравнения (6). Приведем их названия согласно принятой в статье систематизации, обшепринятые названия и s-мультисимволы:

1. Гипергеометрическое уравнение (ГУ) (5) с s-мультисимволом $\{1,1,1\}$.

2. Конфлюэнтное гипергеометрическое уравнение (КГУ) (вырожденное гипергеометрическое уравнение) (7) (см. ниже) с s-мультисимволом $\{1,2\}$.

3. Биконфлюэнтное гипергеометрическое уравнение (БКГУ) (различные формы носят названия: уравнение Вебера, уравнение Эрмита, уравнение параболического цилиндра, уравнение гармонического осциллятора) (8) (см. ниже) с s-мультисимволом $\{3\}$. 
4. Редуцированное конфлюэнтное гипергеометрическое уравнение (РКГУ) (подстановкой $z \mapsto z^{2}$ оно сводится к уравнению Бесселя) (9) (см. ниже) с s-мультисимволом $\{1,3 / 2\}$.

5. Редуцированное биконфлюэнтное гипергеометрическое уравнение (РБКГУ) (уравнение Эйри) (10) (см. ниже) с s-мультисимволом $\{5 / 2\}$.

Приведем эти уравнения, включая в список уравнение (5):

$$
\begin{aligned}
L_{z}^{(1 ; 2)}(a, 1 ; c) y(z) & =\left(z D^{2}+(c-z) D-a\right) y(z)=0, \\
L_{z}^{(; 3)}(a, 1) y(z) & =\left(D^{2}-z D-a\right) y(z)=0, \\
L_{z}^{(1 ; 3 / 2)}(c ; 1) y(z) & =\left(z D^{2}+c D-1\right) y(z)=0, \\
L_{z}^{(1 ; 5 / 2)}(1) y(z) & =\left(D^{2}-z\right) y(z)=0 .
\end{aligned}
$$

Параметры $a, b, c$, фигурируюшие в уравнениях (5), (7)-(10), являются сингулярными параметрами, акцессорных параметров нет, скейлингова параметра тоже нет. Полиномиальные решения для уравнений (5), (7)-(8) возникают при целых неположительных значениях параметра $a$ и совпадают с собственными функциями соответствуюших задач.

\section{5. КЛАСС ГОЙНА}

Перейдем к описанию следующего класса - класса Гойна $(N=4)$ [9-12]. Собственно уравнение Гойна записьвается в виде

$$
\begin{aligned}
& L_{z}^{(1,1,1 ; 1)}(a, b ; s ; d ; t ; \lambda) y(z)=\left(z(z-1)(z-t) D^{2}+(c(z-1)(z-t)+\right. \\
& \quad+d z(z-t)+(a+b+1-c-d) z(z-1)) D+(a b z+\lambda)) y(z)=0 .
\end{aligned}
$$

Локальные характеристики этого уравнения наиболее наглядно могут быть представлены обобщенной схемой Римана

$$
\left(\begin{array}{ccccc}
1 & 1 & 1 & 1 & \\
0 & 1 & t & \infty & ; z \\
0 & 0 & 0 & a & ; \lambda \\
1-c & 1-d & c+d-a-b & b
\end{array}\right)
$$

Как видно из выражений (11), (12), уравнение Гойна характеризуется сингулярными параметрами (безразмерными) $a, b, c, d$, одним скейлинговым параметром (размерным) $t$, задающим положение одной (выделенной) особой точки, и акцессорным (размерным) параметром $\lambda$, имеюшим нелокальный характер и играюшим роль спектрального параметра. В классе Гойна можно выделить 10 типов уравнений. Перечислим их, приведя их названия согласно принятой систематизации, и s-мультисимволы, а ниже - и сами уравнения:

1. Уравнение Гойна (УГ) (11) с s-мультисимволом $\{1,1,1,1\}$.

2. Конфлюэнтное уравнение Гойна (КУГ) (13) с s-мультисимволом $\{1,1,2\}$.

3. Биконфилюэнтное уравнение Гойна (БКУГ) (14) с s-мультисимволом $\{1,3\}$. 
4. Дважды конфлюэнтное уравнение Гойна (ДКУГ) (15) с s-мультисимволом $\{2,2\}$.

5. Триконфлюэнтное уравнение Гойна (ТКУГ) (16) с s-мультисимволом $\{4\}$.

6. Редуцированное конфлюэнтное уравнение Гойна (РКУГ) (17) с s-мультисимволом $\{1,1,3 / 2\}$.

7. Редуцированное биконфлюэнтное уравнение Гойна (РБКУГ) (18) с s-мультисимволом $\{1,5 / 2\}$.

8. Редуцированное дважды конфлюэнтное уравнение Гойна (РДКУГ) (19) с s-мультисимволом $\{3 / 2,2\}$.

9. Дважды редуцированное дважды конфлюэнтное уравнение Гойна (ДР ДКУГ) (20) с s-мультисимволом $\{3 / 2,3 / 2\}$.

10. Редуцированное триконфлюэнтное уравнение Гойна (РТКУГ) (21) с s-мультисимволом $\{7 / 2\}$.

Приведем конфлюэнтные уравнения

$$
\begin{aligned}
L_{z}^{(1,1 ; 2)}(a ; c, d ; t ; \lambda) y(z)= & \left(z(z-1) D^{2}+(-t z(z-1)+c(z-1)+d z) D+\right. \\
& +(-t a z+\lambda)) y(z)=0 \\
L_{z}^{(1 ; 3)}(a ; c ; t ; \lambda) y(z)= & \left(z D^{2}+\left(-z^{2}-t z+c\right) D+(-a z+\lambda)\right) y(z)=0 \\
L_{z}^{(2 ; 2)}(a, c, t ; \lambda) y(z)= & \left(z^{2} D^{2}+\left(-z^{2}+c z-t\right) D+(-a z+\lambda)\right) y(z)=0, \\
L_{z}^{(; 4)}(a ; t ; \lambda) y(z)= & \left(D^{2}+\left(-z^{2}-t\right) D+(-a z+\lambda)\right) y(z)=0
\end{aligned}
$$

и редуцированные конфолюэнтные уравнения

$$
\begin{aligned}
L_{z}^{(1,1 ; 3 / 2)}(c, d, t ; \lambda) y(z)= & \left(z(z-1) D^{2}+(c(z-1)+d z) D+\right. \\
& +(-t z+\lambda)) y(z)=0 \\
L_{z}^{(1 ; 5 / 2)}(c, t ; \lambda) y(z)= & \left(z D^{2}+c D+(-t z+\lambda)\right) y(z)=0, \\
L_{z}^{(3 / 2 ; 2)}(a, t ; \lambda) y(z)= & \left(z^{2} D^{2}-z^{2} D+\left(-a z-\frac{t}{z}+\lambda\right)\right) y(z)=0 \\
L_{z}^{(3 / 2 ; 3 / 2)}(t ; \lambda) y(z)= & \left(z^{2} D^{2}+\left(-z-\frac{t}{z}+\lambda\right)\right) y(z)=0 \\
L_{z}^{(; 7 / 2)}(t ; \lambda) y(z)= & \left(D^{2}+\left(-z^{3}-t z+\lambda\right)\right) y(z)=0,
\end{aligned}
$$

составляющие в дополнение к (11) класс Гойна. Заметим, что дважды редуцированное дважды конфлюэнтное уравнение Гойна заменой независимой переменной (не дробно-линейным преобразованием) преобразуется к частному случаю конффлюэнтного уравнения Гойна.

\section{6. КЛАСС ПЕНЛЕВЕ. СТРУКТУРНАЯ СВЯЗЬ С КЛАССАМИ ЛИНЕЙНЫХ ОДУ}

Уравнениями Пенлеве традиционно называются шесть уравнений, которые известны как $P^{(6)}, P^{(5)}, P^{(4)}, P^{(3)}, P^{(2)}, P^{(1)}$, соответственно. Определенным образом выбран- 
ные решения уравнений Пенлеве - так называемые трансценденты Пенлеве - могут расцениваться как специальные функции нелинейной физики. Решения уравнений Пенлеве характеризуются свойством Пенлеве, а именно, они не могут иметь точек разветвления или существенно особых точек, положение которых зависело бы от начальных данных. Уравнения Пенлеве могут быть получены как условие совместности двух линейных ОДУ (лаксовой пары), первое из которых содержит на одну особенность больше, чем уравнение класса Гойна, причем это ложная регулярная особенность [6], а второе является условием сохранения монодромии решений первого уравнения. Этот факт лежит в основе так называемого метода изомонодромных деформаций. Современное изложение вместо первоначального уравнения второго порядка использует систему первого порядка $2 \times 2$.

Мы введем уравнения Пенлеве нестандартным образом - через структурную связь с классами линейных уравнений. Доказательство того, что стандартные уравнения Пенлеве эквивалентны тем, которые выписаны здесь, выполняется заменой переменных, кроме одного случая, который будет специально обсуждаться. Рассмотрим линейное "квантовое" уравнение

$$
H(q, p, t,) y(q, t)=\lambda y(q, t),
$$

где $q$ - независимая переменная, $p$ - оператор дифференцирования по $q, t$ - скейлинговый параметр и $\lambda$ - акцессорный параметр, играющий роль спектрального параметра. В таком виде записывается каждое уравнение класса Гойна и не могут записываться ни уравнения гипергеометрического класса (нет акцессорного параметра), ни уравнения из классов более сложных, чем класс Гойна (более одного акцессорного параметра).

Для заданного уравнения класса Гойна представление (22) ни в коем случае не единственно. Во-первых, мы можем всегда переопределять спектральный параметр умножением обеих сторон уравнения (22) на произвольную функцию $g(t)$ или добавлением любой функции $g_{1}(t)$ к обеим сторонам (22). Уравнения вида $(22)$ исследуются с точностью до отношений эквивалентности, порожденных s-гомотопными преобразованиями зависимой переменной $y$ и изоморфизмами комплексной независимой переменной $q$. Функция $H(q, p, t)$ в уравнении $(22)$ считается квантовым гамильтонианом.

Для того же самого гамильтониана (22), рассматриваемого как классический объект, можно записать соответствующий лагранжиан $\mathcal{L}(q, \dot{q}, t)$ и уравнения классического движения Эйлера-Лагранжа в виде

$$
\frac{d}{d t} \frac{\partial \mathcal{L}}{\partial \dot{q}}-\frac{\partial \mathcal{L}}{\partial q}=0 \Rightarrow q_{t t}=F\left(q, q_{t}, t\right),
$$

здесь $\dot{q} \equiv q_{t}-$ производная $q$ по $t$. Следует заметить, что параметр $t$ играет в дальнейшем роль времени. Поскольку квантовое уравнение рассматривается без производной по времени и время в гамильтониан входит только параметрически, уравнение (22) следует считать адиабатическим приближением для “истинной” квантовой задачи. Для уравнений класса Пенлеве важными являются критические точки или неподвижные особые точки. В терминах уравнений Гойна это те значения скейлингового параметра $t$, при которых происходит слияние особых точек. 
ТеОрема [13]. Каждое уравнение Пенлеве в виде (23) является уравнением Эйлера-Лагранжа для порождающего его линейного уравнения второго порядка (22), принадлежсащего классу Гойна, и, наоборот, каждое уравнение класса Гойна, записанное в определенной форме, порождает уравнение классического движения, характеризуемое свойством Пенлеве.

Для любого уравнения Гойна гамильтониан в уравнении (22) представим в виде

$$
H(q, p, t)=\frac{1}{f(t)}\left(r_{3}(q, t) p^{2}+r_{2}(q, t) p+r_{1}(q, t)\right) \text {. }
$$

Тогда соответствующий лагранжиан есть

$$
\mathcal{L}=\frac{f(t)}{4 r_{3}(q, t)}\left(q_{t}-\frac{r_{2}(q, t)}{f(t)}\right)^{2}-\frac{r_{1}(q, t)}{f(t)} .
$$

В результате получаются следующие уравнения Эйлера-Лагранжа:

$$
\begin{aligned}
q_{t t} & =\frac{1}{2} \frac{\partial}{\partial q}\left(\ln r_{3}(q, t)\right) q_{t}^{2}-\left(\frac{\partial}{\partial t}(\ln f(t))-\frac{\partial}{\partial t}\left(\ln r_{3}(q, t)\right)\right) q_{t}+ \\
& +\frac{r_{3}(q, t)}{f^{2}(t)}\left(\frac{\partial}{\partial q} \frac{r_{2}^{2}(q, t)}{2 r_{3}(q, t)}+f(t) \frac{\partial}{\partial t} \frac{r_{2}(q, t)}{r_{3}(q, t)}-2 \frac{\partial r_{1}(q, t)}{\partial q}\right)
\end{aligned}
$$

Приведем гамильтонианы нередуцированных уравнений класса Гойна:

УГ-

$$
\begin{aligned}
H^{(1,1,1 ; 1)}(q, p, t)= & -\frac{1}{t(t-1)}\left[q(q-1)(q-t) p^{2}+(c(q-1)(q-t)+\right. \\
& +d q(q-t)+(a+b+1-c-d) q(q-1)) p+a b q]
\end{aligned}
$$

КУГ-

$$
H^{(1,1 ; 2)}(q, p, t)=-\frac{1}{t}\left[q(q-1) p^{2}+(-t q(q-1)+c(q-1)+d q) p-a t q\right],
$$

БКУГ -

$$
H^{(1 ; 3)}(q, p, t)=-\left(q p^{2}+(-q(q+t)+c) p-a q\right)
$$

ДКУГ -

$$
H^{(2 ; 2)}(q, p, t)=-\frac{1}{t}\left(q^{2} p^{2}+\left(-q^{2}-t+c q\right) p-a q\right)
$$

ТКУГ -

$$
H^{(; 4)}(q, p, t)=-\left(p^{2}+\left(-q^{2}-t\right) p-a q\right)
$$

Проделав переход к классической динамике согласно (24)-(26), считая параметр $t$ адиабатическим временем, получим шесть уравнений, которые с точки зрения принятой для класса Гойна терминологии естественно называть уравнением Пенлеве $P^{(1,1,1 ; 1)}$ и конфлюэнтными (нередуцированными !) уравнениями Пенлеве $P^{(1,1 ; 2)}, P^{(1 ; 3)}, P^{(; 4)}$, 
$P^{(2 ; 2)}$. В принятых обозначениях для параметров уравнение Пенлеве $P^{(1,1,1 ; 1)}$, соответствуюшее уравнению Гойна, имеет вид

$$
\begin{aligned}
q_{t t}= & \frac{1}{2}\left(\frac{1}{q}+\frac{1}{q-1}-\frac{1}{q-t}\right) q_{t}^{2}-\left(\frac{1}{t}+\frac{1}{t-1}+\frac{1}{q-t}\right) q_{t}+\frac{q(q-1)(q-t)}{t^{2}(t-1)^{2}} \times \\
& \times\left(\frac{(a+b+1)^{2}}{2}-2 a b-\frac{c^{2} t}{2 q^{2}}+\frac{d^{2}(t-1)}{2(q-1)^{2}}+\frac{\left(1-(c+d-a-b)^{2}\right) t(t-1)}{(q-t)^{2}}\right) .
\end{aligned}
$$

Следующим идет уравнение, которое естественно назвать конфлюэнтным уравнением Пенлеве $P^{(1,1 ; 2)}$,

$$
\begin{aligned}
q_{t t}= & \frac{1}{2}\left(\frac{1}{q}+\frac{1}{q-1}\right) q_{t}^{2}-\frac{1}{t} q_{t}+\frac{1}{t^{2}}\left(\frac{c^{2}(q-1)}{2 q}-\frac{d^{2} q}{2(q-1)}\right)+ \\
& +\frac{q(q-1)(2 q-1)}{2}-\frac{q(q-1)}{t}(1+c+d-2 a) .
\end{aligned}
$$

Далее идут биконфлюэнтное уравнение Пенлеве $P^{(1 ; 3)}$

$$
q_{t t}=\frac{q_{t}^{2}}{2 q}+\frac{3}{2} q^{3}+2 t q^{2}+\left(\frac{t^{2}}{2}+2 a-c\right) q-\frac{c^{2}}{2 q}
$$

дважды конфлюэнтное уравнение Пенлеве $P^{(2 ; 2)}$

$$
q_{t t}=\frac{q_{t}^{2}}{q}-\frac{q_{t}}{t}+\frac{1}{q}+\frac{q^{3}}{t^{2}}+\frac{q^{2}(2 a-c)}{t^{2}}-\frac{c-1}{t}
$$

и триконфлюэнтное уравнение Пенлеве $P^{(; 4)}$

$$
q_{t t}=2 q^{3}+2 t q+2 a .
$$

Следуюший список включает редуцированные уравнения Пенлеве, возникающие по формулам (24)-(26) из редуцированных уравнений Гойна.

Перечислим последние, выписывая только гамильтонианы:

$$
\begin{aligned}
H^{(1,1 ; 3 / 2)}(q, p, t, c, d) & =-\frac{1}{t}\left[q(q-1) p^{2}+(c(q-1)+d q) p-t q\right], \\
H^{(1 ; 5 / 2)}(q, p, t, c) & =-\left(q p^{2}+c p-t q\right), \\
H^{(3 / 2 ; 2)}(q, p, t, a) & =-\frac{1}{t}\left(q^{2} p^{2}-q^{2} p-a q-\frac{t}{q}\right), \\
H^{(3 / 2 ; 3 / 2)}(q, p, t) & =-\frac{1}{t}\left(q^{2} p^{2}-q-\frac{t}{q}\right), \\
H^{(; 7 / 2)}(q, p, t) & =-\left(p^{2}+\left(-q^{3}-t q\right) p\right) .
\end{aligned}
$$

Им отвечают редуцированные уравнения Пенлеве: 


$$
\begin{aligned}
& P^{(1,1 ; 3 / 2)}- \\
& q_{t t}=\frac{1}{2}\left(\frac{1}{q}+\frac{1}{q-1}\right) q_{t}^{2}-\frac{1}{t} q_{t}+\frac{1}{t^{2}}\left(\frac{c^{2}(q-1)}{2 q}-\frac{d^{2} q}{2(q-1)}\right)+\frac{2 q(q-1)}{t}, \\
& P^{(1 ; 5 / 2)}- \\
& q_{t t}=\frac{q_{t}^{2}}{2 q}-\frac{c^{2}}{2 q}+4 q^{2}-2 t q,
\end{aligned}
$$

$P^{(3 / 2 ; 2)}$

$$
q_{t t}=\frac{q_{t}^{2}}{q}-\frac{q_{t}}{t}+\frac{2 a q^{2}+q^{3}}{t^{2}}-\frac{2}{t}
$$

$P^{(3 / 2 ; 3 / 2)}-$

$$
q_{t t}=\frac{q_{t}^{2}}{q}-\frac{q_{t}}{t}+\frac{2 q^{2}}{t^{2}}-\frac{2}{t}
$$

$P^{(; 7 / 2)}-$

$$
q_{t t}=6 q^{2}+t
$$

Уравнение $P^{(; 7 / 2)}$ совпадает с точностью до масштабирования с $P^{(1)}$, т.е. с первым уравнением Пенлеве. Как указал автору Капаев, уравнение $P^{(1 ; 5 / 2)}$ приводится контактным преобразованием ко второму уравнению Пенлеве $P^{(2)}$.

Рассмотрим вопрос о линеаризации уравнений Пенлеве при дополнительном условии $c=0$. Линеаризацию будем понимать в смысле взятия линейной части функции $F\left(q, q_{t}, t\right)$ по переменным $q, q_{t}$, т.е.

$$
\left.\left.F\left(q, q_{t}, t\right)\right|_{c=0} \sim \frac{\left.\partial F\left(q, q_{t}, t\right)\right|_{c=0}}{\partial q_{t}}\right|_{q=0, q_{t}=0} q_{t}+\left.\frac{\left.\partial F\left(q, q_{t}, t\right)\right|_{c=0}}{\partial q}\right|_{q=0, q_{t}=0} q .
$$

Такая линеаризация уравнения Пенлеве $P^{(1,1,1 ; 1)}$ приводит к уравнению, эквивалентному гипергеометрическому,

$$
\begin{aligned}
q_{t t}= & -\frac{q_{t}}{t-1}+\left(\frac{\frac{(a+b+1)^{2}}{2}-2 a b}{(t-1)^{2}}-\frac{\left(1-(d-a-b)^{2}\right)}{t^{2}}+\right. \\
& \left.+\frac{-\frac{(a+b+1)^{2}}{2}+2 a b+\frac{d^{2}}{2}+\left(1-(d-a-b)^{2}\right)}{t(t-1)}\right) q .
\end{aligned}
$$

Линеаризация уравнения Пенлеве $P^{(1,1 ; 2)}$ приводит к уравнению, эквивалентному конфлюэнтному гипергеометрическому уравнению,

$$
q_{t t}=-\frac{q_{t}}{t}+\left(\frac{1}{2}+\frac{1+d-2 a}{t}+\frac{d^{2}}{2 t^{2}}\right) q
$$

Линеаризация уравнения Пенлеве $P^{(1,1 ; 3 / 2)}$ приводит к редуцированному конфлюэнтному гипергеометрическому уравнению

$$
q_{t t}=-\frac{q_{t}}{t}+\left(\frac{d^{2}}{2 t^{2}}+\frac{2}{t}\right) q
$$


Линеаризация уравнения Пенлеве $P^{(1 ; 3)}$ приводит к биконффююэнному гипергеометрическому уравнению

$$
q_{t t}=\left(\frac{t^{2}}{2}+2 a\right) q .
$$

И, наконец, линеаризация уравнений Пенлеве $P^{(; 4)}$ и $P^{(1 ; 5 / 2)}$ приводит к редуцированному биконфлюэнтному гипергеометрическому уравнению

$$
q_{t t}= \pm 2 t q .
$$

Уравнения Пенлеве $P^{(2 ; 2)}, P^{(3 / 2 ; 2)}, P^{(3 / 2 ; 3 / 2)}, P^{(; 7 / 2)}$ не линеаризируются в указанном смысле.

Разобранные структурные связи уравнений гипергеометрического класса, класса Гойна и класса Пенлеве удобно изображать структурными диаграммами вида

$$
\begin{aligned}
\{1,1,1,1\} & \longrightarrow\{1,1,1\} \\
\searrow & \nearrow \\
P^{(6)} & \nearrow
\end{aligned}
$$

ЗАмЕчАниЕ 3. Уравнения Пенлеве можно ввести тремя способами: 1) согласно свойству Пенлеве, 2) как условие совместности соответствуюших лаксовых пар (изомонодромии), 3) как классические аналоги уравнений Гойна. Однако все эти способы вызывают у автора ошущение неудовлетворенности. Первый способ крайне запутан и лишен изяшества. Второй способ требует априорной нормировки решений и неинвариантен по отношению к преобразованиям уравнений. Третий способ также требует априорной нормировки акцессорного параметра и также неинвариантен относительно преобразований уравнения.

\section{7. КОНФЛЮЭНШИИ И “ПРИНЦИП КОНФЛюЭНЦИИ”}

Переход от уравнений, относящихся к одному виду, к уравнениям другого вида осуществляется с помощью следуюших процессов:

1) конфлюэнции,

2) редукиии - уменњшения числа характеризующих уравнение параметров, сопровождаемое уменьшением s-ранга какой-либо особой точки.

Под конфлюэнцией линейного уравнения мы будем понимать предельный переход параметров уравнения, которые параметризованы величиной $\epsilon, \epsilon \rightarrow 0$. Этот переход сопровождается слиянием двух особых точек $z_{1}, z_{2}$ в особую точку $z_{*}$ причем так, чтобы выполнялось условие

$$
R\left(z_{*}\right)>\max \left(R\left(z_{1}\right), R\left(z_{2}\right)\right) .
$$

В случае, если выполняется условие

$$
R\left(z_{*}\right)=R\left(z_{1}\right)+R\left(z_{2}\right),
$$


конфлюэнция называется сильной. В противном случае конфлюэнция называется слабой. Любое из линейных уравнений, рассмотренных выше, можно символически записать в виде

$$
L_{z}^{(\vec{j})} y(z)=0
$$

где $\vec{j}$ является $s$-мультисимволом уравнения. С помошью параметризации коэффициентов уравнения процесс конфлюэнции можно описать символически как

$$
\epsilon^{m} L^{(\vec{j})}(\epsilon) \rightarrow L^{(\vec{k})},
$$

где $L^{(\vec{k})}$ - оператор, полученный в результате конфлюэнции. Решения уравнений $(53)$ удовлетворяют другим равенствам (однородным), записьваемым в виде

$$
T^{(\vec{j})} y(z)=0
$$

Оператор-функция $T^{(\vec{j})}$ должна быть функцией, зависимой от тех же произвольных параметров, что и $L^{(\vec{j})}$, и может тем же способом быть параметризована. Обозначим эту параметризацию через $T^{(\vec{\jmath})}(\epsilon) . T^{(\vec{k})}$ - операторы, полученные в результате конфлюэнции. Тогда в дополнение к уравнениям (53) может быть выполнено соотношение

$$
\epsilon^{m} T^{(\vec{j})}(\epsilon) \rightarrow T^{(\vec{k})}
$$

Исследование свойств каждого из введенных классов уравнений можно строить на базе следующего "принципа конфилюэнции":

пусть $y(z)$ - решение уравнения с исходным оператором, а $\tilde{y}(z)$ - решение уравнения с оператором $L^{(\vec{k})}$. Тогда при определенных дополнительных предположениях функция $\tilde{y}(z)$ является также решением уравнения

$$
T_{z}^{(\vec{k})} y(z)=0
$$

Этот принцип может быть широко применен в различных практических приложениях, что устраняет "хаос" в мире специальных функций. Мы рассмотрим вначале примеры параметризации для реализации процессов конфлюэнции (табл. 2), а затем пример 
применения принципа конфолюэнции.

Таблица 2

Конфлюэнции для гипергеометрического класса

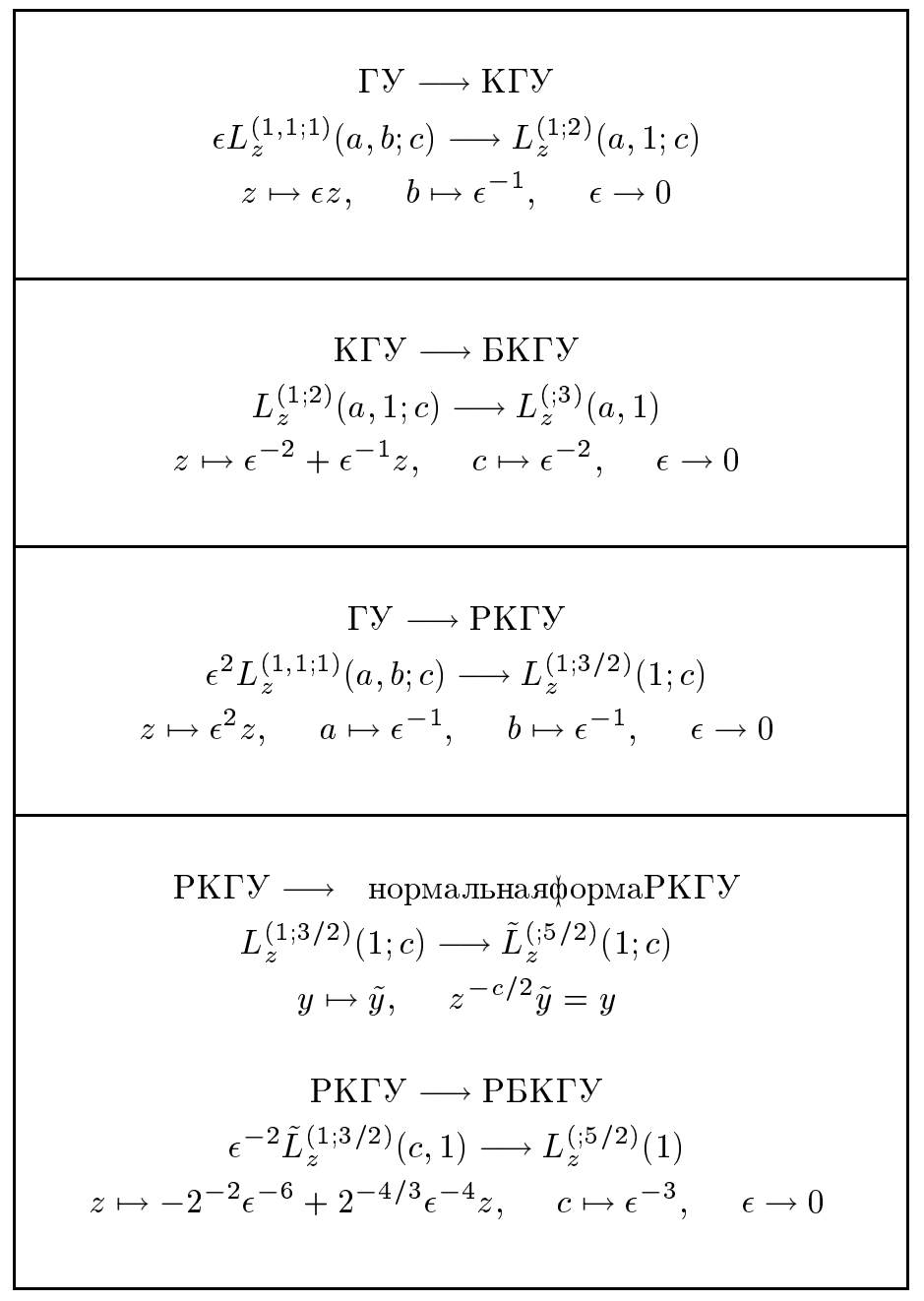

Рассмотренные конфлюэнции могут быть представлены в виде диаграммы

$$
\begin{aligned}
L^{(1,1 ; 1)} & \longrightarrow L^{(1 ; 2)} \longrightarrow L^{(; 3)} \\
& \searrow L^{(1 ; 3 / 2)} \longrightarrow L^{(; 5 / 2)}
\end{aligned}
$$

где горизонтальные стрелки отвечают конфлюэнциям, а наклонная стрелка - слабой конфлюэнщии. 
Таблища 3

Конфлюэнции для уравнений класса Гойна с целыми $s$-мультисимволами

\begin{tabular}{|c|}
\hline $\begin{array}{c}\text { УГ } \longrightarrow \text { КУГ } \\
-\epsilon L_{z}^{(1,1,1 ; 1)}(a, b ; c, d ; t ; \lambda) \rightarrow L_{z}^{(1,1 ; 2)}(a ; c, d ; t ; \lambda) \\
t \mapsto \epsilon^{-1}, \quad b \mapsto p \epsilon^{-1}, \quad \epsilon \rightarrow 0, \quad p:=t\end{array}$ \\
\hline $\begin{array}{c}\text { КУГ } \longrightarrow \text { БКУГ } \\
-\epsilon L_{z}^{(1,1 ; 2)}(a ; c, d ; t ; \lambda) \rightarrow L_{z}^{(1 ; 3)}(a ; c ; t ; \lambda) \\
z \mapsto \epsilon z, \quad t \mapsto-(\epsilon)^{-2}, \quad d \mapsto p \epsilon^{-1}+\epsilon^{-2} \\
\epsilon \rightarrow 0, \quad p:=t\end{array}$ \\
\hline $\begin{array}{c}\text { БКУГ } \longrightarrow \text { ТКУГ } \\
\epsilon L_{z}^{(1 ; 3)}(c, d ; t ; \lambda) \rightarrow L_{z}^{(; 4)}(t ; \lambda) \\
z \mapsto(\epsilon)^{-1} z+(\epsilon)^{-3}, \quad t \mapsto-2(\epsilon)^{-3}, \quad c \mapsto(\epsilon)^{-6}-p(\epsilon)^{-2} \\
\epsilon \rightarrow 0, \quad p:=t\end{array}$ \\
\hline $\begin{array}{c}\text { КУГ } \longrightarrow \text { ДКУГ } \\
-\epsilon L_{z}^{(1,1 ; 2)}(a ; c, d ; t ; \lambda) \rightarrow L_{z}^{(2 ; 2)}(a ; c ; t ; \lambda) \\
z \mapsto(\epsilon)^{-1} z, \quad t \mapsto \epsilon, \quad d \mapsto p(\epsilon)^{-1}, \quad c \mapsto c-p(\epsilon)^{-1} \\
\epsilon \rightarrow 0, \quad p:=t\end{array}$ \\
\hline
\end{tabular}

В табл. 3 не включен предельный переход для $\lambda$. Слабые конфлюэнции для класса Гойна требуют при своем описании больших подробностей и здесь не приведены.

В качестве примера применения принципа конфлюэнции рассмотрим вывод интегрального представления для конфлюэнтной гипергеометрической функции из интегрального представления для гипергеометрической функции. Пусть параметры гипергеометрического уравнения удовлетворяют условиям $\operatorname{Re} c>\operatorname{Re} b>0$. Тогда регулярное в точке $z=0$ решение уравнения (5) - гипергеометрическая функция $F(a, b, c ; z)-$ имеет интегральное представление

$$
F(a, b, c ; z)=B \int_{0}^{1}(1-t z)^{-a}(1-t)^{c-b-1} t^{b-1} d t
$$

с соответствуюшим множителем $B$, определяемым нормировкой функции. 
Из представления (58) путем конфлюэнции согласно табл. 1 получим интегральное представление

$$
\Phi(a, c ; z)=D \int_{0}^{1} e^{z t} t^{a-1}(1-t)^{c-a-1} d t
$$

для регулярного в нуле решения конфолюэнтного гипергеометрического уравнения (7) конфлюэнтной гипергеометрической функции $\Phi(a, c ; z)$.

Благодарности. Автор благодарен А. Зеегеру и В. Лаю за ценные дискуссии на предыдущем этапе работы, А. Капаеву за ссылку на уравнение для $P^{(1 ; 5 / 2)}$, А. Шабату за просмотр первой редакции статьи и А. Акопяну за изготовление графических элементов текста. Работа была поддержана грантами РФФИ 96-01-00-668 и 98-01-01-075, а также грантом DFG.

\section{Список литературы}

[1] Ф. Олвер. Асимптотика и специальные функции. М.: Наука, 1990.

[2] Г. Бейтмен, А. Эрдейи. Высшие трансцендентные функции. В трех томах. М.: Наука, 1965, $1966,1967$.

[3] E. L. Ince. Ordinary Differential Equations. New York: Dover Publications, 1959.

[4] E. T. Whittaker, G. N. Watson. A Course of Modern Analysis. Cambridge: Cambridge University Press, 1927.

[5] P. Moon, D. E. Spencer. Field Theory Handbook. Berlin-Heidelberg-New York: Springer, 1971.

[6] K. Iwasaki, H. Kimura, S. Shimomura, M. Ioshida. From Gauss to Painleve: a Modern Theory of Special Functions. Braunschweig: Vieweg, 1991.

[7] Р. Г. Баранцев. Дефиниция асимптотики и системные триады. В сб.: Асимптотические методы в теории систем. Гл. ред. А.Н. Панченков. Иркутск: СО АН СССР, 1980. С. 70-81.

[8] А. Зеегер, В. Лай, С. Ю. Славянов. ТМФ. 1995. Т. 104. № 2. С. 233-247.

[9] Centennial Workshop on Heun's Equation - Theory and Applications. Eds. A. Seeger, W. Lay. Stuttgart: MPI/MF, 1990.

[10] A. Decarreau, M. C. Dumont-Lepage, P. Maroni, A. Robert and A. Ronveaux. Ann. Soc. Sc. Bruxelles. 1978. V. 92. P. 53-78.

[11] A. Decarreau, P. Maroni and A. Robert. Ann. Soc. Sc. Bruxelles. 1978. V. 92. P. 151-189.

[12] The Heun's Differential Equation. Ed. A. Ronveaux. Oxford: Oxford University Press, 1995.

[13] S. Yu. Slavyanov. J. Phys. A. 1996. V. 29. P. 7329-7335.

Поступила в редакцию 9.VII.1998 г., после доработки 23.XI.1998 г. 